\title{
Oxidative Stress in Cardiovascular Inflammation: Its Involvement in Autoimmune Responses
}

\author{
Elisabetta Profumo, Brigitta Buttari, and Rachele Riganò \\ Dipartimento di Malattie Infettive, Parassitarie ed Immunomediate, Istituto Superiore di Sanità, Viale Regina Elena 299, \\ 00161 Roma, Italy \\ Correspondence should be addressed to Elisabetta Profumo, elisabetta.profumo@iss.it
}

Received 1 April 2011; Accepted 3 May 2011

Academic Editor: Adrian Chester

Copyright (๑) 2011 Elisabetta Profumo et al. This is an open access article distributed under the Creative Commons Attribution License, which permits unrestricted use, distribution, and reproduction in any medium, provided the original work is properly cited.

\begin{abstract}
Recently, it has become clear that atherosclerosis is a chronic inflammatory disease in which inflammation and immune responses play a key role. Accelerated atherosclerosis has been reported in patients with autoimmune diseases, suggesting an involvement of autoimmune mechanisms in atherogenesis. Different self-antigens or modified self-molecules have been identified as target of humoral and cellular immune responses in patients with atherosclerotic disease. Oxidative stress, increasingly reported in these patients, is the major event causing structural modification of proteins with consequent appearance of neoepitopes. Self-molecules modified by oxidative events can become targets of autoimmune reactions, thus sustaining the inflammatory mechanisms involved in endothelial dysfunction and plaque development. In this paper, we will summarize the best characterized autoantigens in atherosclerosis and their possible role in cardiovascular inflammation.
\end{abstract}

\section{Introduction}

Atherosclerosis was initially believed as a process caused by the passive accumulation of lipids in the vessel wall. Recently, however, it has become clear that atherosclerosis is a complex condition and that multiple pathogenic factors contribute to trigger and sustain vessel wall damage [1]. Experimental studies have clearly demonstrated that atherosclerosis can be considered a chronic inflammatory disease of the arterial wall and that inflammation plays a key role in all stages of the pathogenic process, including formation, progression, and rupture of atherosclerotic plaque [2,3]. In early lesions, chemokines and adhesion molecules expressed by endothelial cells recruit inflammatory cells, in particular monocytes and $\mathrm{T}$ cells, to the arterial intima [4]. If the acute inflammatory response does not resolve and macrophages and $\mathrm{T}$ cells continue to accumulate in the intima, a fibrotic repair process activates, leading to the formation of an atherosclerotic lesion [5]. This process is driven by the inflammatory cells that secrete cytokines and growth factors and stimulate smooth muscle cells to migrate to the intima, where they proliferate and produce extracellular matrix proteins [4]. In the advanced lesion, matrix metalloproteinases secreted from activated macrophages degrade the connective tissue in the fibrous cap causing plaque rupture.

Accelerated atherosclerosis has been reported in patients with various autoimmune diseases, suggesting an involvement of autoimmune mechanisms in atherogenesis [6-9]. This phenomenon is attributed to the presence of traditional risk factors for atherosclerosis, but could be also the result of autoimmune and inflammatory mechanisms that are aggravated in these diseases [6].

The first evidence for the involvement of autoimmune responses in atherosclerosis was provided by Jonasson et al. [10] who demonstrated the expression of MHC class II molecules and the presence of activated $T$ cells in human atherosclerotic plaques. Analysis of plaque-infiltrating $\mathrm{T}$ cells revealed that these cells primarily showed the proinflammatory Th1 phenotype [11]. Subsequent studies on different animal models demonstrated that activation of Th1 responses contributes to a more aggressive progression of atherosclerosis [4]. Different studies have strongly suggested that atherosclerosis to some extent can be viewed as an autoimmune disease in which the adaptive immune system 
is targeted against self-antigens modified by biochemical factors such as oxidative stress and hypercholesterolemia [12]. In this respect, atherosclerosis shares some similarities with other diseases characterized by an autoimmune etiology, such as type I diabetes and rheumatoid arthritis. In this contest, identifying the antigens responsible for the activation of immune responses that promote and sustain inflammation in atherosclerosis may be relevant.

\section{Mechanisms of Autoimmunity Generation}

Although infectious agents have been associated with the activation of immune mechanisms, evidence exist that the main antigenic targets in atherosclerosis are modified endogenous structures [12]. Atherosclerotic plaques express autoantigens that are targeted by both IgM and IgG. It is likely that these autoimmune responses initially have a beneficial effect facilitating the removal of potentially harmful antigens [13]. However, studies performed on hypercholesterolaemic mice deficient in different components of innate and adaptive immunity uniformly indicate that the net effect of immune activation is proatherogenic $[13,14]$ and that atherosclerosis, at least to some extent, should be regarded as an autoimmune disease.

In general, three mechanisms have been proposed to explain the autoimmune phenomena. (i) The molecular mimicry theory proposes that the autoimmune response is triggered by the initial activation of lymphocytes recognizing epitopes derived from microorganisms that share sequence homology with self-molecules. Therefore, autoimmunity resulting from epitope mimicry may be a consequence of the antipathogen immune response that can lead to disease in predisposed individuals [15]. (ii) Impairments in apoptosis execution and clearance can potentially render apoptotic cells as a source of autoantigens normally sequestered in the intracellular environment. Structural changes occurring during cell death may influence the immunogenicity of clustered self-antigens at the surface of apoptotic bodies [16]. (iii) Autoimmune responses may be directed against selfstructures altered by high-affinity ligand binding or by chemical damage due to environmental events, such as oxidative stress [17].

\section{Oxidative Stress in Atherosclerosis}

Excessive oxidative stress and chronic inflammation are both major characteristics of atherosclerosis [18, 19]. However, the mechanisms by which oxidative stress mediates cardiovascular diseases are not clear [20]. Oxidative stress is the major event causing protein structural modification and appearance of neo-/cryptic epitopes [21,22]. Several systems that generate reactive oxygen species (ROS) catalyze a variety of oxidative damage to nucleic acids, lipids, and proteins [23]. In physiological conditions oxidative stress is well compensated. When there is an overproduction of ROS or a deficiency of antioxidant enzyme activity, a biological damage with vascular lesion formation and functional defects may occur [24].
Oxidative stress and inflammation may determine the modification of self-structures also favouring the formation of advanced glycation end products (AGEs). Chronic oxidative stress causes an accumulation of AGEs [25]. The generation of AGEs and augmentation of proinflammatory mechanisms in the vessel provide a potent feedback loop for sustained oxidant stress, ongoing generation of AGEs, and vascular perturbation.

Epidemiological studies have demonstrated an inverse association between risk of stroke and intake of several antioxidant-rich food items $[26,27]$. Intake of high total antioxidant capacity (the cumulative capacity of food components to scavenge free radicals) has been related to reduced inflammation and increased circulating antioxidants in both cross-sectional and randomized intervention studies $[28,29]$. Nevertheless, clinical trials with anti-oxidants in humans have shown conflicting results in protecting against detrimental cardiovascular outcomes [20]. In particular, most antioxidant vitamin trials have failed to reduce cardiovascular morbidity and mortality [30]. In contrast, other investigators sustain that the beneficial effects of lipidlowering and antihypertensive treatments are in part due to their antioxidant properties [31, 32]. Furthermore, recent findings suggest that antioxidants may play a role in reducing the risk of cerebral infarction [33], thus confirming the pathogenetic role of oxidative stress in inflammation and cardiovascular pathology. Collectively, these studies suggest that more rigorous clinical trial designs are needed to evaluate the usefulness of an antioxidant-based therapeutic approach to cardiovascular diseases [34].

\section{Autoantigens Implicated in Cardiovascular Diseases}

4.1. Oxidized Low-Density Lipoproteins. Oxidized low-density lipoproteins (LDLs) is the best characterized autoantigen in atherosclerosis. LDL modifications caused by oxidation and enzymatic attacks result in the release of proinflammatory phospholipids and lipid peroxides, which rapidly activate an inflammatory response in surrounding cells $[35,36]$. The oxidation process is also associated with major structural modifications of LDL determining the formation of new antigenic epitopes which can be presented by dendritic cells and give rise to clonal expansion of oxidized LDL-specific $\mathrm{T}$ cells [12]. About $10 \%$ of all $\mathrm{T}$ cells present in human atherosclerotic plaques specifically recognize oxidized LDL [17], and these cells are also present in the circulation [37]. Furthermore, autoantibodies specific for oxidized LDL have been detected in humans and associated with cardiovascular disease, but their role is controversial $[38,39]$. Pilot studies in animal models have provided promising results for the development of vaccines based on oxidized LDL antigens [12].

4.2. Heat Shock Proteins. A second category of autoantigens that have been implicated in atherosclerosis are the stressinduced heat shock proteins (HSPs) [40]. HSPs act as molecular chaperons facilitating refolding of denatured proteins 
in stressed cells. Interestingly, some of them have also been implicated in loading of immunogenic peptides to major histocompatibility class (MHC) I and II molecules [41]. Besides being expressed in cells under physiological conditions, HSPs increase in response to many environmental stresses, including oxidative stress [42]. Under stress conditions, HSPs are expressed not only within cells, but also on the cell surface and can be released into the intercellular space. Intracellular HSPs have cytoprotective functions whereas extracellular located or membrane-bound HSPs mediate immunological functions [43]. In atherosclerotic lesions, human HSPs appear to stimulate an immune response leading to the development and progression of atherosclerosis [44, 45]. Antibody levels against HSP-60/65 are increased in subjects with established cardiovascular disease and predict further development of the disease [46]. These antibodies specifically react with cells in atherosclerotic plaques and mediate lysis of stressed endothelial cells and macrophages in vitro [47]. Recently, we demonstrated that human HSP90 is overexpressed in plaque and serum from patients with carotid atherosclerosis and induces humoral and cellular immune responses in these patients, implicating this self-protein as a possible target autoantigen in the pathogenesis of carotid atherosclerosis $[48,49]$. In particular, we detected HSP90specific $\mathrm{T}$ lymphocytes within the atherosclerotic plaque; these cells showed a predominant Th1, pro-inflammatory profile, suggesting a role for HSP90 in sustaining the inflammatory mechanisms responsible for the thrombogenicity of the atherosclerotic lesion [49]. Major questions for future research are the possible role of circulating anti-HSP90 antibodies and perhaps HSP90-specific peripheral blood T cells as diagnostic and prognostic markers of disease.

4.3. Beta2-Glycoprotein I. Beta2-glycoprotein I ( $\beta 2-\mathrm{GPI})$ is a plasma protein involved in the haemostatic system that has been detected in carotid atherosclerotic lesions. Previous studies in animal models demonstrated that the transfer of lymphocytes obtained from $\beta 2$-GPI-immunized mice was associated with the presence of larger fatty streaks within the recipients compared with animals that received lymphocytes from control mice, suggesting that $\mathrm{T}$ cells specific for $\beta 2-\mathrm{GPI}$ are capable to increase atherosclerosis and that $\beta 2-\mathrm{GPI}$ is a target autoantigen in this disease [6].

$\beta 2-\mathrm{GPI}$ is also the most common target for antiphospholipid antibodies (aPLs). These autoantibodies are associated with thrombotic events and with the incidence of accelerated atherosclerosis in patients with antiphospholipid antibody syndrome and systemic lupus erythematosus [50-52]. In a previous study, we observed that $\beta 2-\mathrm{GPI}$, as several plasma proteins involved in the haemostatic system, undergoes major structural and functional changes upon exposure to oxidative stress and that such modification renders this self-molecule able to activate immature monocyte-derived dendritic cells (DCs) [21]. We demonstrated that DCs stimulated with the oxidized form of human $\beta 2$-GPI presented a mature phenotype, produced cytokines that support T-cell activation, and were able to activate a Th1-type response by allogenic naïve $\mathrm{T}$ cells, characterized by interferon (IFN) $-\gamma$ production. These findings are in accordance with our recent study demonstrating that $\beta 2-\mathrm{GPI}$ is a target antigen of Th1 cellular immune response in patients with carotid atherosclerosis [53]. In this study, we used the native form of the plasma protein, but our previous evidence that $\beta 2$ GPI spontaneously undergoes oxidative modification in in vitro culture conditions [21] led us to hypothesize that T-cell reactivity detected in these patients is directed to the oxidized form of the protein.

More recently, we have demonstrated that a glycated form of $\beta 2$-GPI is able to activate monocyte-derived immature DCs through RAGE engagement and to trigger a proinflammatory signaling pathway mediated by the activation of mitogen-activated protein kinases and nuclear factor- $\kappa \mathrm{B}$ [54].

Our findings underline the role of glycation/glycoxidation in rendering this self-protein able to activate the immune system and suggest that chronic activation of autoimmune reactions against $\beta 2$-GPI modified by oxidative events may contribute to local and systemic inflammation, thus sustaining endothelial dysfunction and promoting thrombotic events in patients with cardiovascular diseases.

4.4. Oxidized Haemoglobin. Microvascular haemorrhage in atherosclerotic plaques is a common event in advanced lesions [55]. Intraplaque haemorrhage is an event characterizing high-risk "vulnerable" plaques, which are prone to rupture and associated with acute thrombotic events. Intraplaque haemorrhage causes the deposition of blood products into the extravascular space, particularly red blood cells (RBCs) and haemoglobin ( $\mathrm{Hb})$. In physiological conditions, $\mathrm{Hb}$ released within the vascular compartment from destroyed RBCs dimerizes and is rapidly bound by the serum protein haptoglobin. The haptoglobin- $\mathrm{Hb}$ complex is recognized by the scavenger receptor CD163 on the surface of mononuclear phagocytes, that promotes endocytosis and degradation of the complex [56]. Complicated plaques are characterized by repetitive haemorrhage events and hemolysis, accompanied with the release of large amounts of $\mathrm{Hb}$ into the atherosclerotic lesions. In these conditions, the capacity of $\mathrm{Hb}$ scavenging mechanisms is saturated. The proinflammatory and pro-oxidant microenvironment within the plaque may predispose free $\mathrm{Hb}$ to oxidative modifications. Free $\mathrm{Hb}$ or its oxidized forms may represent dangerous autoantigens, unwanted target of immune responses initially directed to the removal of escaped or modified proteins. Following this hypothesis, we have examined the presence of $\mathrm{T}$ cells specific for oxidized $\mathrm{Hb}$ in the peripheral blood of patients with advanced carotid atherosclerosis [57]. We used an oxidized $\mathrm{Hb}$ preparation predominantly containing hemichromes. Hemichromes represent $\mathrm{Hb}$ oxidation products characterized by a cross-linking between globin and heme that is responsible for molecule distortion and structural changes. We observed that the mean frequency of IFN- $\gamma$-secreting $\mathrm{T}$ lymphocytes specific for oxidized $\mathrm{Hb}$ was significantly higher in patients with carotid atherosclerosis than in healthy subjects, suggesting that the oxidized forms of $\mathrm{Hb}$ display immunodominant $\mathrm{T}$-cell epitopes that are able 
to stimulate adaptive immunity. Furthermore, our oxidized $\mathrm{Hb}$ preparation enhanced phenotypic maturation of LPSstimulated DCs, as demonstrated by the appearance of the DC maturation marker CD83 and by the upregulation of the costimulatory molecules CD80, CD40, HLA-DR, and CD86, and significantly increased the production of the proinflammatory cytokines interleukin (IL)-12p70 and tumor necrosis factor (TNF)- $\alpha$ by DCs [57]. In a more recent study, we extended our results and demonstrated the presence of $\mathrm{T}$ lymphocytes specific for oxidized $\mathrm{Hb}$ within human atherosclerotic plaques. These cells produced high levels of the pro-inflammatory Th1 cytokines IFN- $\gamma$ and TNF- $\alpha$ [58].

Previous studies demonstrated that $\mathrm{Hb}$ exerts a proinflammatory and oxidative action on endothelial cells [59, 60]. In particular, oxidized $\mathrm{Hb}$ upregulates the transcription rate of several pro-inflammatory genes, that is, E-selectin, ICAM-1, and VCAM-1, on vascular endothelial cells through the activation of the NF- $\kappa \mathrm{B}$ family of transcription factors [61]. Our data implicate oxidized $\mathrm{Hb}$ as a possible antigenic target of cell-mediated immune responses contributing to tissue damage in the pathogenesis of carotid atherosclerosis. We can hypothesize that intraplaque haemorrhage in complicated lesions determines the release of large amounts of free $\mathrm{Hb}$. In these conditions, haptoglobin and CD163, getting overwhelmed, are unable to clear efficiently the protein which accumulates in the extracellular compartment $[55,62]$. The pro-oxidant microenvironment within the atherosclerotic plaque may promote oxidative modifications of $\mathrm{Hb}$ determining the generation of novel antigenic epitopes on this self-molecule. These epitopes can activate specific $\mathrm{T}$ lymphocytes with a predominant Th1 profile that contribute to inflammation. In physiological conditions, the oxidized forms of $\mathrm{Hb}$ do not reach consistent levels in vivo because oxidative stress is well compensated. Of note, we observed the presence of $\mathrm{T}$-cells specific for oxidized $\mathrm{Hb}$ also in the peripheral blood from healthy subjects, suggesting that $\mathrm{T}$ cell clones specific for this rare molecule are not deleted within the thymus as, in basal conditions, they do not encounter the target antigen necessary for their activation.

Overall, these results indicate that $\mathrm{Hb}$, in particular conditions, can sustain endothelial dysfunction interacting with endothelial cells and/or with immune cells, thus promoting the pathogenetic mechanisms involved in the progression of cardiovascular diseases.

\section{Conclusions}

Excessive oxidative stress and low-grade chronic inflammation are major pathophysiological factors contributing to the development of cardiovascular diseases. The strong oxidative and inflammatory conditions occurring in patients with atherosclerosis trigger the generation of a series of oxidation byproducts that may contribute to the pathogenesis of the disease. In addition to pro-inflammatory properties, self molecules modified by oxidative events can become targets of autoimmune reactions, thus sustaining the inflammatory mechanisms involved in endothelial dysfunction and plaque development (Figure 1). More studies aimed to clarify these

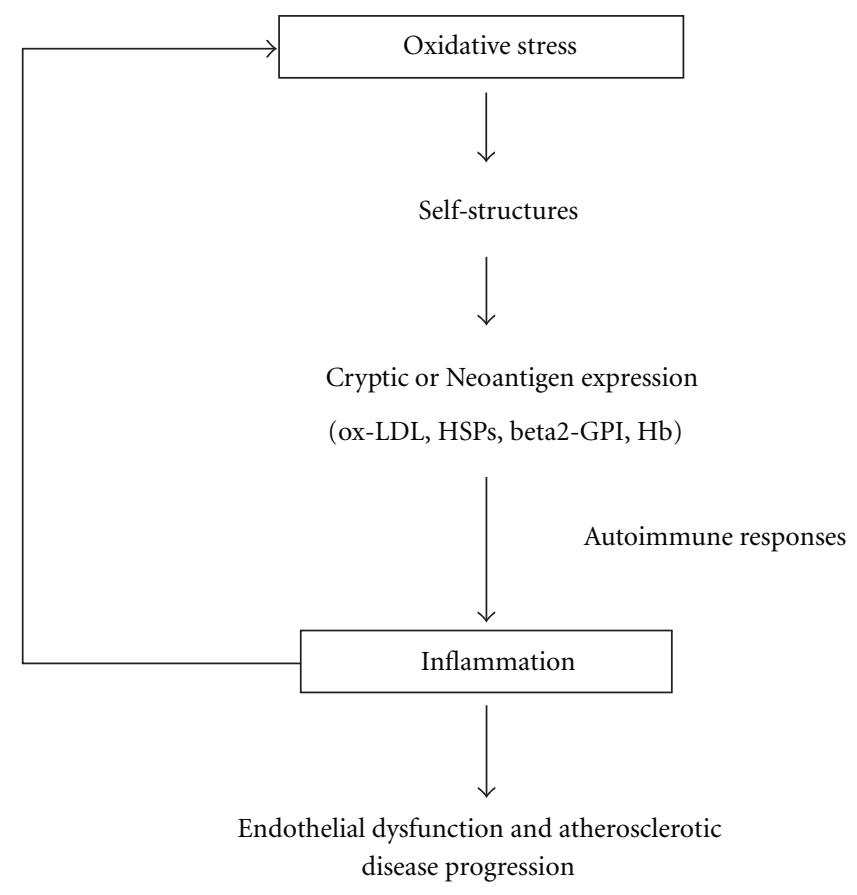

FIGURE 1: Schematic representation of the relationship between oxidative stress and inflammation in cardiovascular diseases. Patients with atherosclerosis are characterized by strong oxidative stress conditions. Prooxidant compounds that form may interact with molecular and cellular components thus determining expression of cryptic antigens, such as HSPs, and/or structural modifications of self-molecules and generation of neoepitopes, such as oxidized LDL. These cryptic or neoantigens may trigger autoimmune reactions, thus sustaining the inflammatory mechanisms involved in endothelial dysfunction and plaque progression. Of note, molecular and cellular mediators of inflammatory mechanisms sustain oxidative stress, thus creating a pathogenic system that amplifies itself. Ox-LDL: oxidized low-density lipoproteins; HSPs: heat shock proteins; beta2-GPI: beta2-glycoprotein I; Hb: haemoglobin.

aspect might be useful for designing novel preventive strategies in cardiovascular diseases. Modulation of the immune system could represent a useful approach to prevent and/or treat these diseases. A vaccination approach might be a useful, effective tool in the modern arsenal of cardiovascular therapy and could possibly be used on a large scale at a low cost. Several modalities of vaccines have been tested against oxidized LDL, $\beta 2$-GPI, HSPs, cholesterol, and other molecules, with promising results. Nevertheless, a deeper understanding of the role of immunization in atherosclerosis will be essential to the use of vaccines in clinical medicine $[63,64]$.

\section{References}

[1] E. Bartoloni, Y. Shoenfeld, and R. Gerli, "Inflammatory and autoimmune mechanisms in the induction of atherosclerotic damage in systemic rheumatic diseases: two faces of the same coin," Arthritis Care and Research, vol. 63, no. 2, pp. 178-183, 2011. 
[2] P. Libby, Y. Okamoto, V. Z. Rocha, and E. Folco, "Inflammation in atherosclerosis: transition from theory to practice," Circulation Journal, vol. 74, no. 2, pp. 213-220, 2010.

[3] S. I. van Leuven, R. Franssen, J. J. Kastelein, M. Levi, E. S. G. Stroes, and P. P. Tak, "Systemic inflammation as a risk factor for atherothrombosis," Rheumatology, vol. 47, no. 1, pp. 3-7, 2008.

[4] J. Nilsson, M. Wigren, and P. K. Shah, "Regulatory T cells and the control of modified lipoprotein autoimmunity-driven atherosclerosis," Trends in Cardiovascular Medicine, vol. 19, no. 8, pp. 272-276, 2009.

[5] G. K. Hansson, "Mechanisms of disease: inflammation, atherosclerosis, and coronary artery disease," New England Journal of Medicine, vol. 352, no. 16, pp. 1685-1626, 2005.

[6] Y. Shoenfeld, R. Gerli, A. Doria et al., "Accelerated atherosclerosis in autoimmune rheumatic diseases," Circulation, vol. 112, no. 21, pp. 3337-3347, 2005.

[7] N. Bassi, A. Ghirardello, L. Iaccarino et al., "OxLDL/ $\beta$ GPIanti-oxLDL/ $\beta$ GPI complex and atherosclerosis in SLE patients," Autoimmunity Reviews, vol. 7, no. 1, pp. 52-58, 2007.

[8] H. Zinger, Y. Sherer, and Y. Shoenfeld, "Atherosclerosis in autoimmune rheumatic diseases-mechanisms and clinical findings.," Clinical Reviews in Allergy \& Immunology, vol. 37, no. 1, pp. 20-28, 2009.

[9] E. Matsuura, K. Kobayashi, and L. R. Lopez, "Preventing autoimmune and infection triggered atherosclerosis for an enduring healthful lifestyle," Autoimmunity Reviews, vol. 7, no. 3, pp. 214-222, 2008.

[10] L. Jonasson, J. Holm, and O. Skalli, "Expression of class II transplantation antigen on vascular smooth muscle cells in human atherosclerosis," Journal of Clinical Investigation, vol. 76, no. 1, pp. 125-131, 1985.

[11] J. Frostegård, A. K. Ulfgren, P. Nyberg et al., "Cytokine expression in advanced human atherosclerotic plaques: dominance of pro-inflammatory (Th1) and macrophage-stimulating cytokines," Atherosclerosis, vol. 145, no. 1, pp. 33-43, 1999.

[12] J. Nilsson and G. K. Hansson, "Autoimmunity in atherosclerosis: a protective response losing control?" Journal of Internal Medicine, vol. 263, no. 5, pp. 464-478, 2008.

[13] G. K. Hansson and P. Libby, "The immune response in atherosclerosis: a double-edged sword," Nature Reviews Immunology, vol. 6, no. 7, pp. 508-519, 2006.

[14] C. J. Binder, M. K. Chang, P. X. Shaw et al., "Innate and acquired immunity in atherogenesis," Nature Medicine, vol. 8, no. 11, pp. 1218-1226, 2002.

[15] M. Blank and Y. Shoenfeld, "Beta-2-glycoprotein-I, infections, antiphospholipid syndrome and therapeutic considerations," Clinical Immunology, vol. 112, no. 2, pp. 190-199, 2004.

[16] J. C. Hall, L. Casciola-Rosen, and A. Rosen, "Altered structure of autoantigens during apoptosis," Rheumatic Disease Clinics of North America, vol. 30, no. 3, pp. 455-471, 2004.

[17] S. Stemme, B. Faber, J. Holm, O. Wiklund, J. L. Witztum, and G. K. Hansson, "T lymphocytes from human atherosclerotic plaques recognize oxidized low density lipoprotein," Proceedings of the National Academy of Sciences of the United States of America, vol. 92, no. 9, pp. 3893-3897, 1995.

[18] S. Lee, Y. Park, M. Y. Zuidema, M. Hannink, and C. Zhang, "Effects of interventions on oxidative stress and inflammation of cardiovascular diseases," World Journal of Cardiology, vol. 3, no. 1, pp. 18-24, 2011.

[19] N. R. Madamanchi, A. Vendrov, and M. S. Runge, "Oxidative stress and vascular disease," Arteriosclerosis, Thrombosis, and Vascular Biology, vol. 25, no. 1, pp. 29-38, 2005.
[20] J. C. Sartori-Valinotti, R. Iliescu, L. A. Fortepiani, L. L. Yanes, and J. F. Reckelhoff, "Sex differences in oxidative stress and the impact on blood pressure control and cardiovascular disease," Clinical and Experimental Pharmacology and Physiology, vol. 34, no. 9, pp. 938-945, 2007.

[21] B. Buttari, E. Profumo, V. Mattei et al., "Oxidized $\beta$-glycoprotein I induces human dendritic cell maturation and promotes a T helper type 1 response," Blood, vol. 106, no. 12, pp. 3880-3887, 2005.

[22] T. W. Stief, J. U. Kurz, M. O. Doss, and J. Fareed, "Singlet oxygen inactivates fibrinogen, factor V, factor VIII, factor X, and platelet aggregation of human blood," Thrombosis Research, vol. 97, no. 6, pp. 473-480, 2000.

[23] J. Arvieux, V. Regnault, E. Hachulla, L. Darnige, F. Berthou, and P. Youinou, "Oxidation of $\beta 2$-glycoprotein I ( $\beta 2-\mathrm{GPI})$ by the hydroxil radical alters phospholipid binding and modulates recognition by- $\beta 2$-GPI autoantibodies," Thrombosis and Haemostasis, vol. 86, no. 4, pp. 1070-1076, 2001.

[24] P. Puddu, G. M. Puddu, E. Cravero, S. De Pascalis, and A. Muscari, "The emerging role of cardiovascular risk factorinduced mitochondrial dysfunction in atherogenesis," Journal of Biomedical Science, vol. 16, p. 112, 2009.

[25] R. Ramasamy, S. F. Yan, and A. M. Schmidt, "RAGE: therapeutic target and biomarker of the inflammatory response- the evidence mounts," Journal of Leukocyte Biology, vol. 86, no. 3, pp. 505-512, 2009.

[26] L. Dauchet, P. Amouyel, and J. Dallongeville, "Fruit and vegetable consumption and risk of stroke: a meta-analysis of cohort studies," Neurology, vol. 65, no. 8, pp. 1193-1197, 2005.

[27] Z. Vokó, M. Hollander, A. Hofman, P. J. Koudstaal, and M. M. B. Breteler, "Dietary antioxidants and the risk of ischemic stroke: the Rotterdam Study," Neurology, vol. 61, no. 9, pp. 1273-1275, 2003.

[28] F. Brighenti, S. Valtueña, N. Pellegrini et al., "Total antioxidant capacity of the diet is inversely and independently related to plasma concentration of high-sensitivity C-reactive protein in adult Italian subjects," British Journal of Nutrition, vol. 93, no. 5, pp. 619-625, 2005.

[29] S. Valtueña, N. Pellegrini, L. Franzini et al., "Food selection based on total antioxidant capacity can modify antioxidant intake, systemic inflammation, and liver function without altering markers of oxidative stress," American Journal of Clinical Nutrition, vol. 87, no. 5, pp. 1290-1297, 2008.

[30] R. Brigelius-Flohé, F. J. Kelly, J. T. Salonen, J. Neuzil, J. M. Zingg, and A. Azzi, "The European perspective on vitamin E: current knowledge and future research," American Journal of Clinical Nutrition, vol. 76, no. 4, pp. 703-716, 2002.

[31] E. M. Lonn, S. Yusuf, V. Dzavik et al., "Effects of ramipril and vitamin E on atherosclerosis: the study to evaluate carotid ultrasound changes in patients treated with Ramipril and vitamin E (SECURE)," Circulation, vol. 103, no. 7, pp. 919 925, 2001.

[32] B. Dahlöf, R. B. Devereux, S. E. Kjeldsen et al., "Cardiovascular morbidity and mortality in the Losartan Intervention For Endpoint reduction in hypertension study (LIFE): a randomised trial against atenolol," Lancet, vol. 359, no. 9311, pp. 995-1003, 2002.

[33] D. Del Rio, C. Agnoli, N. Pellegrini et al., "Total antioxidant capacity of the diet is associated with lower risk of ischemic stroke in a large italian cohort," Journal of Nutrition, vol. 141, no. 1, pp. 118-123, 2011.

[34] M. J. Thomson, V. Puntmann, and J. C. Kaski, "Atherosclerosis and oxidant stress: the end of the road for antioxidant vitamin 
treatment?" Cardiovascular Drugs and Therapy, vol. 21, no. 3, pp. 195-210, 2007.

[35] C. K. Glass and J. L. Witztum, "Atherosclerosis: the road ahead," Cell, vol. 104, no. 4, pp. 503-516, 2001.

[36] N. Leitinger, "Oxidized phospholipids as modulators of inflammation in atherosclerosis," Current Opinion in Lipidology, vol. 14, no. 5, pp. 421-430, 2003.

[37] J. Frostegard, R. Wu, R. Giscombe, G. Holm, A. K. Lefvert, and J. Nilsson, "Induction of T-cell activation by oxidized low density lipoprotein," Arteriosclerosis and Thrombosis, vol. 12, no. 4, pp. 461-467, 1992.

[38] J. Hulthe, "Antibodies to oxidized LDL in atherosclerosis development-clinical and animal studies," Clinica Chimica Acta, vol. 348, no. 1-2, pp. 1-8, 2004.

[39] J. Nilsson and P. T. Kovanen, "Will autoantibodies help to determine severity and progression of atherosclerosis?" Current Opinion in Lipidology, vol. 15, no. 5, pp. 499-503, 2004.

[40] G. Wick, M. Knoflach, and Q. Xu, "Autoimmune and inflammatory mechanisms in atherosclerosis," Annual Review of Immunology, vol. 22, pp. 361-403, 2004.

[41] B. Javid, P. A. MacAry, and P. J. Lehner, "Structure and function: heat shock proteins and adaptive immunity," Journal of Immunology, vol. 179, no. 4, pp. 2035-2040, 2007.

[42] T. A. Mehta, J. Greenman, C. Ettelaie, A. Venkatasubramaniam, I. C. Chetter, and P. T. McCollum, "Heat shock proteins in vascular disease-a review," European Journal of Vascular and Endovascular Surgery, vol. 29, no. 4, pp. 395-402, 2005.

[43] M. F. Tsan and B. Gao, "Heat shock protein and innate immunity," Cellular \& Molecular Immunology, vol. 1, no. 4, pp. 274-279, 2004.

[44] A. G. Pockley, "Heat shock proteins, inflammation, and cardiovascular disease," Circulation, vol. 105, no. 8, pp. 1012 1017, 2002.

[45] H. K. Park, E. C. Park, S. W. Bae et al., "Expression of heat shock protein 27 in human atherosclerotic plaques and increased plasma level of heat shock protein 27 in patients with acute coronary syndrome," Circulation, vol. 114, no. 9, pp. 886-893, 2006.

[46] Q. Xu, S. Kiechl, M. Mayr et al., "Association of serum antibodies to heat-shock protein 65 with carotid atherosclerosis: clinical significance determined in a follow-up study," Circulation, vol. 100, no. 11, pp. 1169-1174, 1999.

[47] M. Mayr, B. Metzler, S. Kiechl et al., "Endothelial cytotoxicity mediated by serum antibodies to heat shock proteins of Escherichia coli and Chlamydia pneumoniae: immune reactions to heat shock proteins as a possible link between infection and atherosclerosis," Circulation, vol. 99, no. 12, pp. 1560-1566, 1999.

[48] R. Riganò, E. Profumo, B. Buttari et al., "Heat shock proteins and autoimmunity in patients with carotid atherosclerosis," Annals of the New York Academy of Sciences, vol. 1107, pp. 110, 2007.

[49] R. Businaro, E. Profumo, A. Tagliani et al., "Heat-shock protein 90: a novel autoantigen in human carotid atherosclerosis," Atherosclerosis, vol. 207, no. 1, pp. 74-83, 2009.

[50] M. L. Bertolaccini, G. R. V. Hughes, and M. A. Khamashta, "Revisiting antiphospholipid antibodies: from targeting phospholipids to phospholipid binding proteins," Clinical Laboratory, vol. 50, no. 11-12, pp. 653-665, 2004.

[51] J. S. Levine, W. Branch, and J. Rauch, "The antiphospholipid syndrome," New England Journal of Medicine, vol. 346, no. 10, pp. $752-763,2002$.
[52] E. Matsuura and L. R. Lopez, "Autoimmune-mediated atherothrombosis," Lupus, vol. 17, no. 10, pp. 878-887, 2008.

[53] E. Profumo, B. Buttari, C. Alessandri et al., "Beta2-glycoprotein $\mathrm{I}$ is a target of $\mathrm{t}$ cell reactivity in patients with advanced carotid atherosclerotic plaques," International Journal of Immunopathology and Pharmacology, vol. 23, no. 1, pp. 73-80, 2010.

[54] B. Buttari, E. Profumo, A. Capozzi, F. Facchiano, L. Saso, and M. Sorice, "Advanced glycation end products of human beta2glycoproteinI modulate the maturation and function of DCs," Blood, vol. 117, no. 23, pp. 6152-6161, 2011.

[55] A. P. Levy and P. R. Moreno, "Intraplaque hemorrhage," Current Molecular Medicine, vol. 6, no. 5, pp. 479-488, 2006.

[56] M. Kristiansen, J. H. Graversen, C. Jacobsen et al., "Identification of the haemoglobin scavenger receptor," Nature, vol. 409, no. 6817, pp. 198-201, 2001.

[57] B. Buttari, E. Profumo, L. Petrone et al., "Free hemoglobin: a dangerous signal for the immune system in patients with carotid atherosclerosis?" Annals of the New York Academy of Sciences, vol. 1107, pp. 42-50, 2007.

[58] E. Profumo, B. Buttari, and R. Riganò, "Oxidized haemoglobin as antigenic target of cell-mediated immune reactions in patients with carotid atherosclerosis," Autoimmunity Reviews, vol. 8, no. 7, pp. 558-562, 2009.

[59] P. W. Buehler, B. Abraham, F. Vallelian et al., "Haptoglobin preserves the CD163 hemoglobin scavenger pathway by shielding hemoglobin from peroxidative modification," Blood, vol. 113, no. 11, pp. 2578-2586, 2009.

[60] X. Liu and Z. Spolarics, "Methemoglobin is a potent activator of endothelial cells by stimulating IL- 6 and IL-8 production and E-selectin membrane expression," American Journal of Physiology, vol. 285, no. 5, pp. C1036-C1046, 2003.

[61] G. Silva, V. Jeney, A. Chora, R. Larsen, J. Balla, and M. P. Soares, "Oxidized hemoglobin is an endogenous proinflammatory agonist that targets vascular endothelial cells," Journal of Biological Chemistry, vol. 284, no. 43, pp. 29582-29595, 2009.

[62] R. P. Rother, L. Bell, P. Hillmen, and M. T. Gladwin, "The clinical sequelae of intravascular hemolysis and extracellular plasma hemoglobin: a novel mechanism of human disease," Journal of the American Medical Association, vol. 293, no. 13, pp. 1653-1662, 2005.

[63] J. F. Carvalho, R. M. R. Pereira, and Y. Shoenfeld, "Vaccination, atherosclerosis and systemic lupus erythematosus," Lupus, vol. 18, no. 13, pp. 1209-1212, 2009.

[64] J. F. De Carvalho, R. M. R. Pereira, and Y. Shoenfeld, "Vaccination for atherosclerosis," Clinical Reviews in Allergy and Immunology, vol. 38, no. 2-3, pp. 135-140, 2010. 


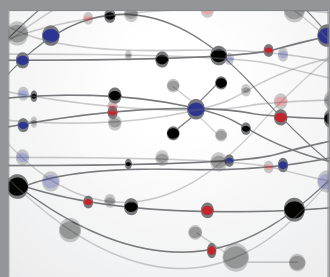

The Scientific World Journal
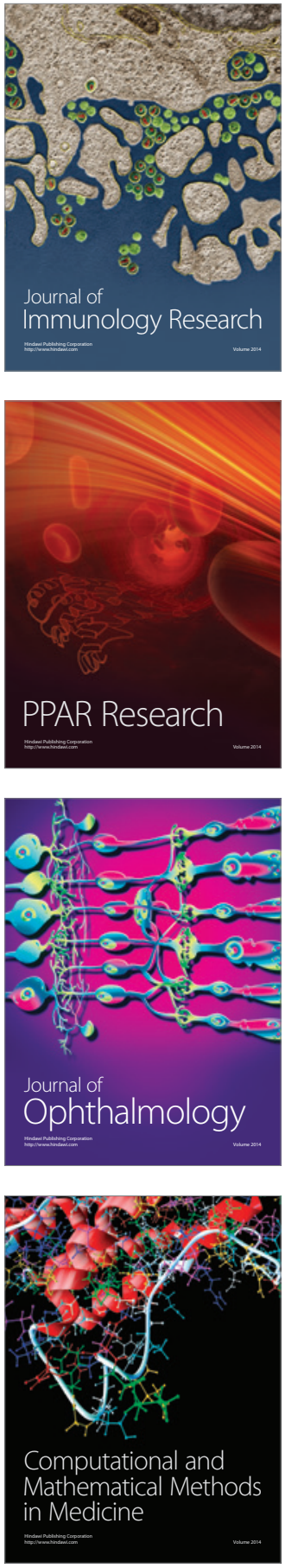

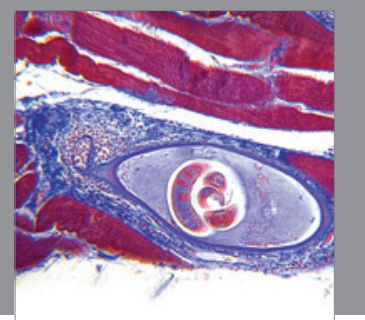

Gastroenterology

Research and Practice
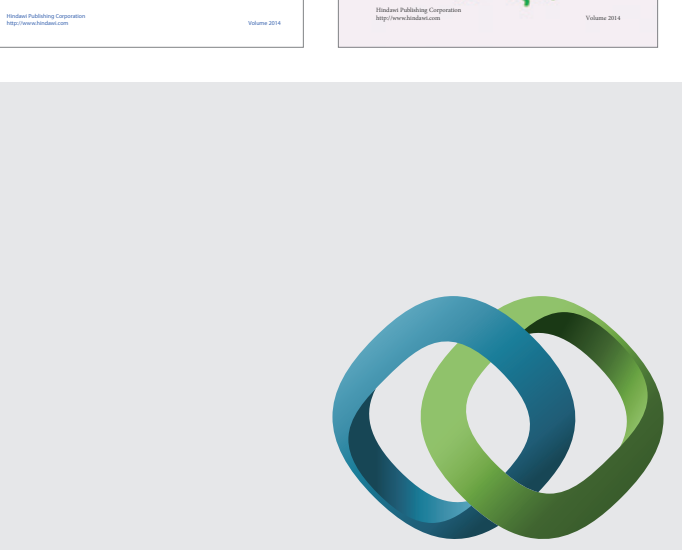

\section{Hindawi}

Submit your manuscripts at

http://www.hindawi.com
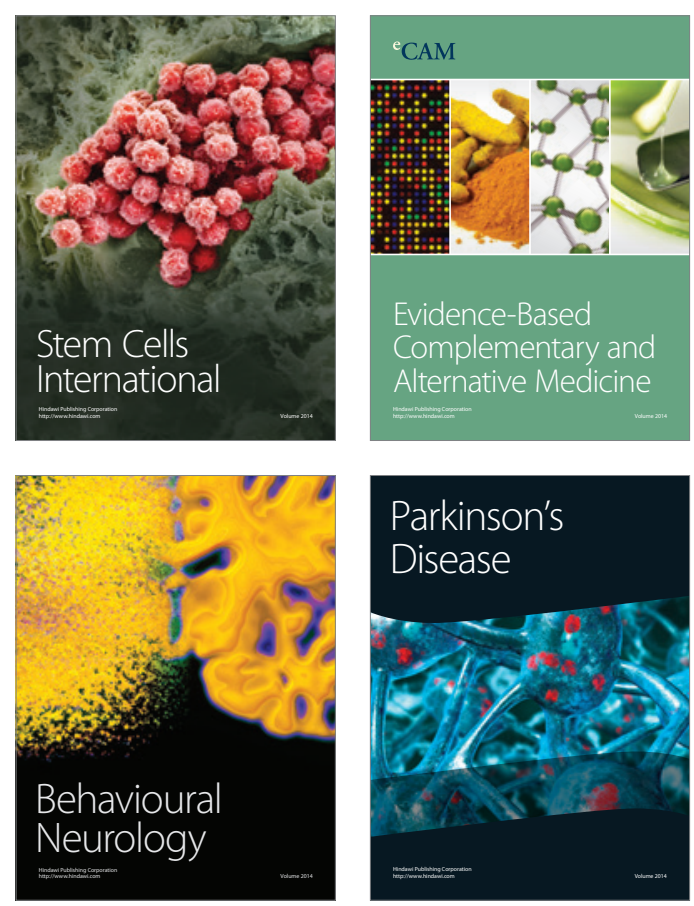

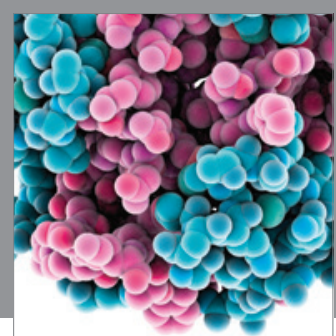

Journal of
Diabetes Research

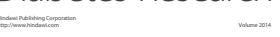

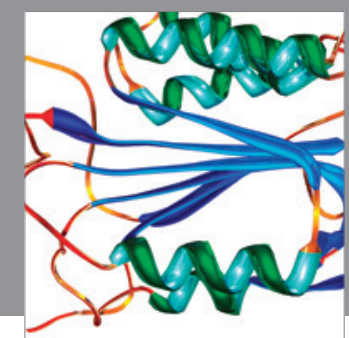

Disease Markers
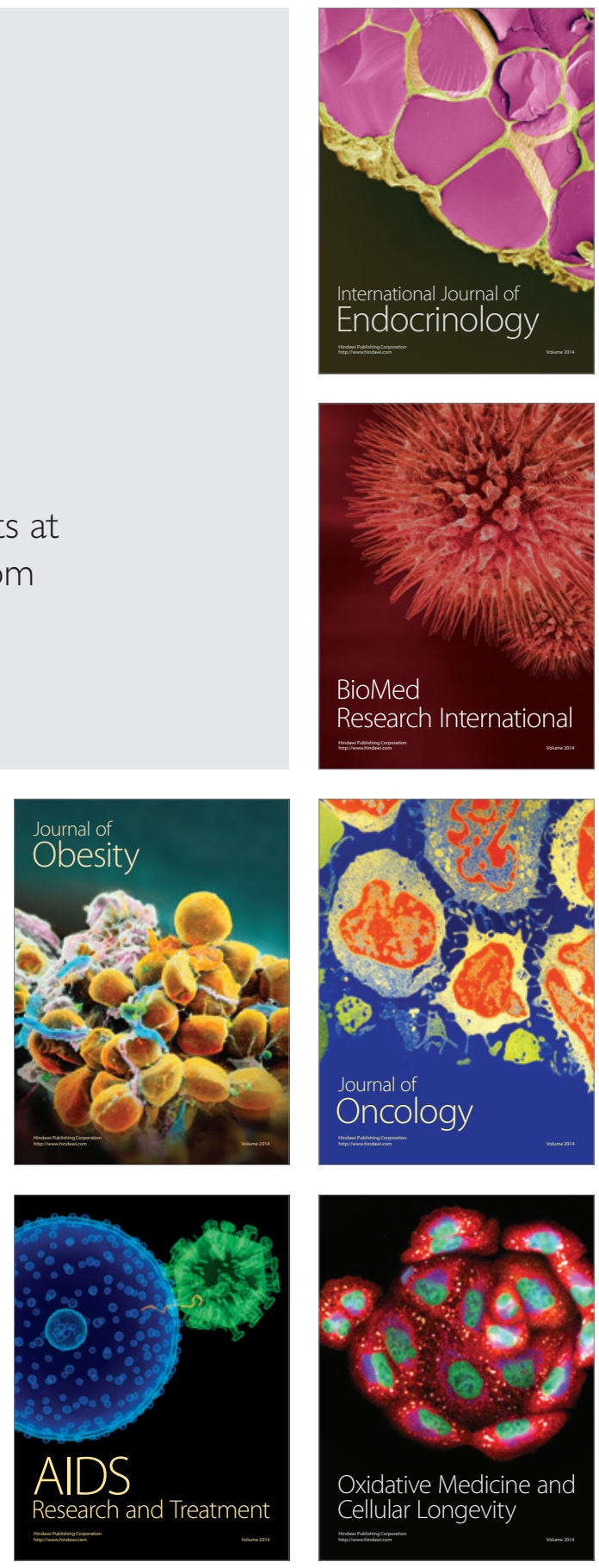